\title{
Construcción del Observatorio Geomagnético de Mérida, Venezuela
}

\author{
Edwin Camacho* \\ José Serra* \\ María Muñiz $^{* *}$ \\ José Gandarilla*
}

Recibido en mayo de 2013; aceptado en diciembre de 2013

\begin{abstract}
A new geomagnetic observatory has been built on Mérida, Venezuela $\left(8^{\circ} 33^{\prime} \mathrm{N}\right.$, $\left.71^{\circ} 19^{\prime} \mathrm{O}, 1755 \mathrm{~m}\right)$. The observatory consists of three wood houses free of magnetic materials, two houses of 3 by 3 meters and one of 3 by 6 meters, also has two external pillars, these facilities are already built. The observatory site is far enough from man-made disturbances and has an horizontal and vertical gradient of less than $1 \mathrm{nT} / \mathrm{m}$. the whole construction has been carried out following the recommendations stated in International Association of Geomagnetism and Aeronomy (IAGA) "Guide for magnetic measurements and observatory practice". It's worthy of remark that there is 180 geomagnetic observatories worldwide of which only 15 are in Latin America.
\end{abstract}

Key words: geomagnetism, observatory.

\section{Resumen}

Un nuevo observatorio geomagnético se ha construido en Mérida, Venezuela $\left(8^{\circ} 33^{\prime} \mathrm{N}, 71^{\circ} 19^{\prime} \mathrm{O}, 1755 \mathrm{~m}\right)$, siendo este observatorio el primero en nuestro país. El emplazamiento del observatorio se encuentra alejado de las perturbaciones provocadas por el hombre (ruido cultural), del mismo modo, se cuenta con un área apropiada para su correcto funcionamiento; obteniéndose un gradiente horizontal y vertical menor a $1 \mathrm{nT} / \mathrm{m}$, esto es excelente para la operatividad del mismo. Toda la construcción se ha llevado a cabo bajo las recomendaciones de la Asociación Internacional de Geomagnetismo y Aeronomía (IAGA, por sus siglas en inglés) mediante el libro de Jankowski y Sucksdorff (1996). El observatorio está conformado por tres casas o pabellones de madera y dos pilares externos libres de materiales magnéti-

* Centro de Investigaciones de Astronomía "Francisco J. Duarte", Venezuela.

** Instituto de Geofísica y Astronomía, Cuba. 
cos. Estas casas son construidas para albergar los equipos de medición denominados "magnetómetros", los cuales están constituidos por equipos de alta sensibilidad para las mediciones del campo geomagnético.

Palabras clave: geomagnetismo, observatorios.

\section{Introducción}

Los observatorios geomagnéticos son centros científicos o laboratorios, encargados de registrar de manera continua y precisa como mínimo tres componentes del vector campo geomagnético " $\mathrm{F}$ " como una función del tiempo, con una resolución de un minuto o menos. Las componentes registradas usualmente son la declinación " $\mathrm{D}$ ", inclinación "I" y el módulo de "F". Uno de los objetivos de los observatorios es proveer la información más precisa sobre la variación secular y variaciones con escalas temporales que van desde minutos y/o segundos hasta décadas. El tiempo de registro es referido al tiempo universal (UT, por sus siglas en inglés).

Un observatorio geomagnético debe poseer las siguientes características: mediciones continuas de las componentes del campo en periodos de un minuto como mínimo, se debe mantener en el mismo lugar de medición con el pasar de los años y publicar periódicamente los datos definitivos (filtrados) a la comunidad científica (St-Louis B. y Sauter E., 2004).

Actualmente existen alrededor de 180 observatorios geomagnéticos en el mundo, pero solo cerca de 15 observatorios se encuentran operativos en América Latina. En Venezuela la construcción del primer observatorio geomagnético fue realizada en el marco de los proyectos de colaboración Cuba-Venezuela, el ente ejecutor del proyecto fue el Centro de Investigaciones de Astronomía "Francisco J. Duarte" (CIDA) que tiene su sede en Mérida, Venezuela; y se contó con la asesoría del personal del Instituto de Geofísica y Astronomía (IGA) de Cuba. Este proyecto tiene como objetivo general:

- La construcción del primer observatorio geomagnético en la República Bolivariana de Venezuela

Pero para alcanzar dicha meta se fijaron los siguientes objetivos especificos:

- Determinar el emplazamiento para el observatorio geomagnético

- Comprar equipos de medición (magnetómetros)

- Diseñar y construir tres pequeños casas o pabellones que conforman el observatorio geomagnético. 
El proyecto para la construcción del observatorio fue realizado siguiendo las recomendaciones de la IAGA, con el propósito de que este nuevo observatorio forme parte de la Red Internacional de Observatorios Magnéticos (INTERMAGNET).

\section{Emplazamiento del observatorio geomagnético}

Cuando se planifica la construcción de un observatorio conviene tener en consideración que este debe funcionar el mayor tiempo posible en ese lugar de emplazamiento. Una de sus principales tareas es realizar un seguimiento de la variación secular del campo geomagnético, y para esto diez o incluso cien años es considerado un tiempo corto. Por otro lado, se debe tener en cuenta que el lugar seleccionado deber ser apropiado magnéticamente para realizar las mediciones del campo, es decir, en las cercanías del observatorio no debe haber fuentes de perturbaciones magnéticas naturales o artificiales, debido a que perjudica la calidad de los datos registrados. Por estas razones las propiedades magnéticas de la zona prevista para el observatorio deben ser estudias cuidadosamente.

Entre los requisitos que debe cumplir el emplazamiento, para la construcción de un nuevo observatorio geomagnético (Jankowski y Sucksdorff, 1996), se tienen:

- Homogeneidad magnética en los terrenos del emplazamiento, los cambios en las propiedades magnéticas en los alrededores del observatorio no son aceptables. Para esto se realiza una exploración o prospección magnética con magnetómetros en toda el área destinada para el observatorio y dicha área debe tener gradientes magnéticos horizontales y verticales menores a un $\mathrm{nT} / \mathrm{m}$ (nano-Tesla sobre metro). Grandes anomalías magnéticas (cientos de nT) no debe ser aceptado cerca de la zona del observatorio.

- El subsuelo de la zona del observatorio debe tener una conductividad eléctrica homogénea, para no tener corrientes inducidas en las rocas de la corteza terrestre, que puedan afectar una o varias componentes del campo (Gubbins y Herrero, 2007). Las costas, es uno de los lugares donde mejor se pueden apreciar los cambios de la conductividad eléctrica y de hecho en los observatorios, se pueden registrar un fenómeno denominado "Efecto costa", por esta razón se recomienda que los observatorios geomagnéticos se construyan a decenas de kilómetros lejos de las costas.

- Un espacio reservado para el observatorio, lo que significa un radio aproximado $300 \mathrm{~m}$ medios desde la casa de mediciones absolutas.

- Alejados de centros poblados, zonas industriales, antenas de comunicación, y cualquier construcción metálica y líneas de alta tensión para evitar el ruido cultural. 
Para seleccionar la ubicación del primer observatorio de Venezuela se realizaron estudios en cuatro terrenos candidatos (Camacho et al., 2009) siendo el más apropiado para el emplazamiento del observatorio los terrenos del Instituto Venezolano de Investigaciones Científicas (IVIC) sede Jají, Estado Mérida, Venezuela. Los terrenos del IVIC-Mérida resultaron favorables para el emplazamiento del observatorio porque presentaron las siguientes características:

1. Se obtuvo que los terrenos presentan gradientes magnéticos verticales y horizontales son menores de un nano Tesla sobre metro (nT/m), y siendo su geología rocas sedimentarias de formación la Quinta. La prospección fue realizada con dos magnetómetros protónicos portátiles como es recomendado (Wienert, 1970), uno fue empleado para el levantamiento geomagnético y el otro como estación base, para posteriormente realizar la corrección por la variación diurna a los datos del levantamiento. La prospección fue realizada sobre todo el terreno destinado para el observatorio geomagnético, realizando mediciones con el magnetómetro cada 10m. En la Figura 1 se muestran los gradientes del terreno destinado para el observatorio, las " $\mathrm{X}$ " muestran los lugares donde fueron construidos los pabellones que conforman el observatorio.

2. Además de presentar gradientes magnéticas favorables, los terrenos del IVICMérida se encuentran lo suficientemente alejados (véase Figura 2) de perturbaciones hechas por el hombre como: edificaciones, carreteras, líneas eléctricas, puentes, entre otros, y abarca un espacio lo suficientemente grande, necesario para el correcto funcionamiento del observatorio.

Aparte de los estudios habituales para la construcción de un observatorio, se hizo un estudio geotécnico en los terrenos del IVIC-Mérida, donde se realizaron exploraciones mediante perforaciones y en los cuales se reportaron los siguientes datos: longitud de perforación, columna estratigráfica, número de golpes/pies correspondientes a la prueba de penetración (STP), entre otros; de los cuales se realizaron ensayos de laboratorios como: contenido de humedad, granulometría por tamizado, límites de consistencias, entre otros. En dicho estudio fue recomendado construir fundaciones superficiales de tipo aislada considerando la topografía inclinada, la geología, la humedad del terreno sobre la que se implantarán los pabellones.

\section{Equipos de medición presentes en el observatorio}

Los instrumentos de medición del campo geomagnético del observatorio de Mérida fueron escogidos con el propósito de cumplir con los estándares internacionales en cuanto a precisión ( $1 \mathrm{nT})$, frecuencia de muestreo (cada minuto o menos), resolución 




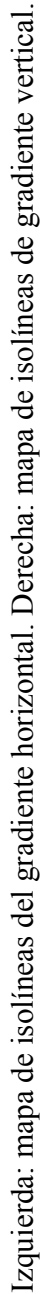

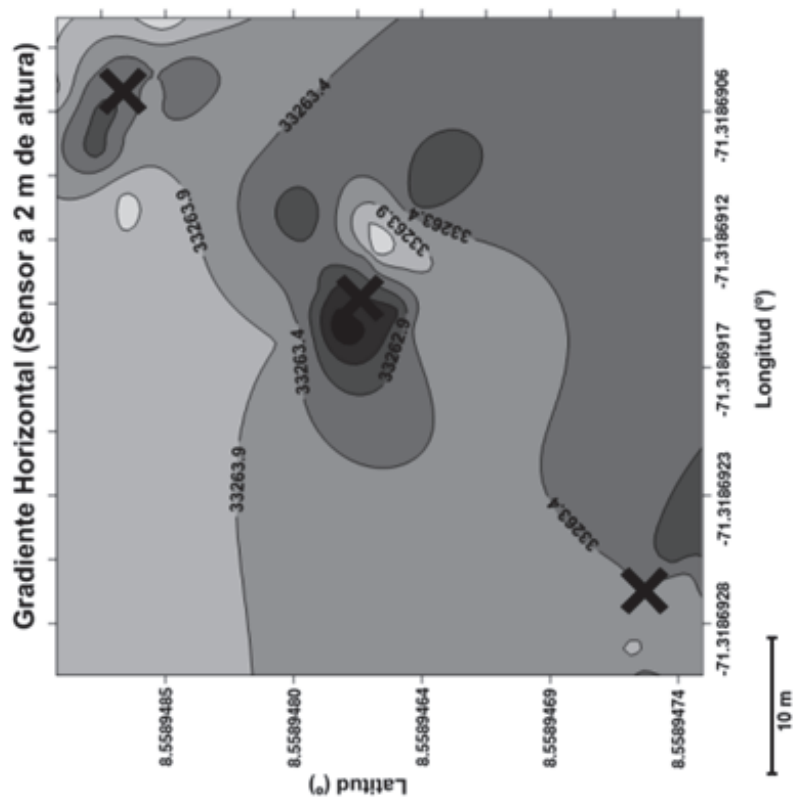

$\underset{5}{\stackrel{5}{50}}$ 


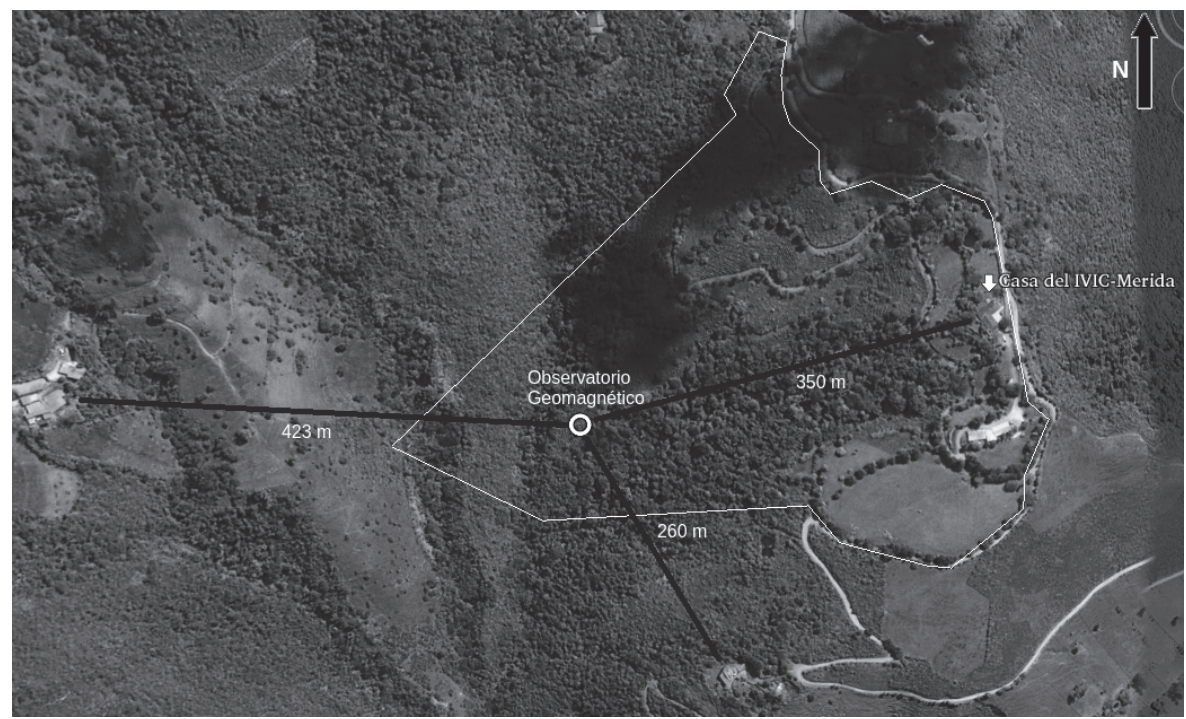

Figura 2. Mapas donde se muestra los linderos de los terrenos del IVIC-Mérida, y las distancias de posibles perturbaciones magnéticas (ruido cultural) que pueden afectar los datos del observatorio geomagnético.

(0.1nT) y calidad de los datos medidos, con la finalidad que este nuevo observatorio forme parte de la red INTERMAGNET. Los magnetómetros adquiridos son:

- Bartington Mag-01H, consiste en un teodolito no magnético con sensor Fluxgate. Usado para medir la declinación e inclinación magnética.

- GEM Systems. GSM-90FD5, variómetro que mide fluctuaciones de las componentes: inclinación "I", declinación " $D$ " y la intensidad total "F" del campo geomagnético. Este equipo por si solo permite realizar observaciones de la magnitud y dirección del campo geomagnético.

- GemSystems GSM-90 EUROMAG, magnetómetro escalar de precesión de protones de efecto Overhauser. Permite obtener la intensidad total del campo geomagnético.

- GemSystems GSM-19T, magnetómetro escalar portátil, que permite realizar prospección magnética. Contiene un sistema de GPS incorporado al equipo que permite obtener las coordenadas geográficas donde son realizadas las medicines y el tiempo en UT. 


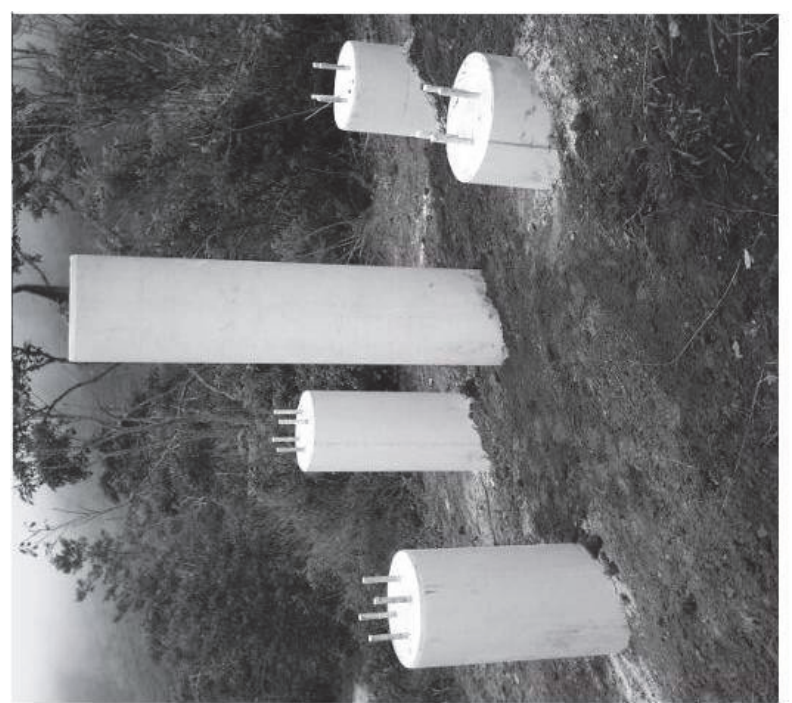

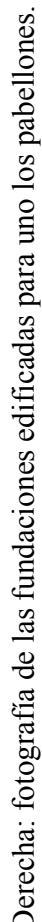

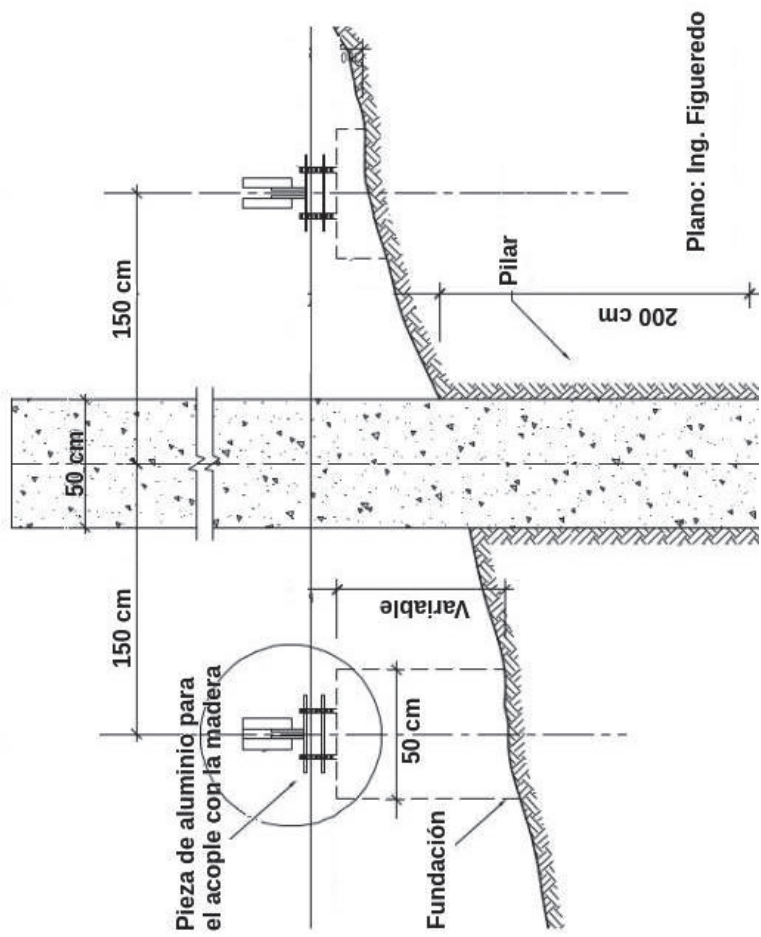




\section{Diseño y construcción del observatorio}

Para este observatorio se contempló la construcción de fundaciones aisladas para cada pabellón o caseta, con un pilar en el centro, como se muestra en la Figura 3. Los pilares son estructuras verticales donde son ubicados los equipos de medición y deben ser muy estables aún y con el pasar de los años, para lo cual fueron enterradas dos metros por debajo del nivel del suelo.

Los pabellones fueron diseñados para ser construidos con madera. La madera empleada para la elaboración de los pabellones fue Pino Caribe y estuvo sometida a un tratamiento previo en el Laboratorio Nacional de Productos Forestales de la Universidad de los Andes, con el propósito de preservar la madera de insectos y alargar la vida útil de la misma. Para ensamblar los pabellones se propuso un método constructivo que permitiera articular los mismos con gran facilidad, en los cuales se usaron tarugos y ensambles de uniones madera-madera, por lo cual fueron empleados pocos clavos de aleación de latón y bronce. En la Figura 4 se muestran planos de dos de los pabellones de madera que conforman el observatorio de Mérida.

Todos los pabellones de madera fueron diseñados con doble techo para mantener la temperatura estable dentro de los mismos, esto es uno de los requisitos al momento de diseñar un observatorio geomagnético. Los cambios de temperatura pueden afectar la precisión de los instrumentos de medición. En la Figura 5 y la Figura 6 se muestran los tres pabellones de madera que conforman el observatorio. Cada pabellón fue diseñado para albergar un equipo de medición, por ende, cada uno tiene un equipo asignado.

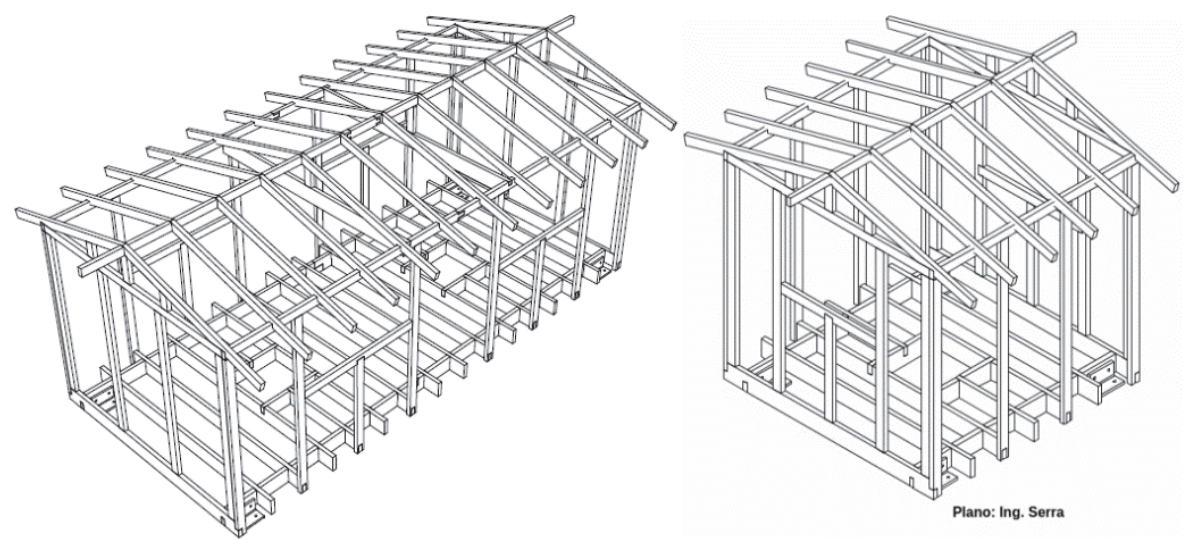

Figura 4. Izquierda: plano de pabellón de madera de dimensiones 3x6m. Derecha: plano de pabellón de madera de dimensiones $3 \times 3 \mathrm{~m}$. 

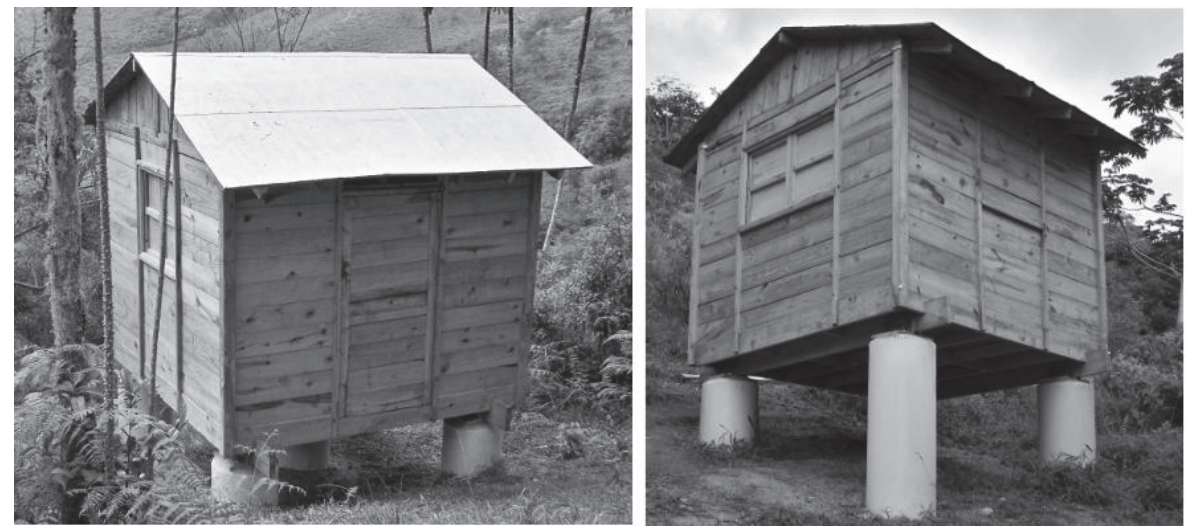

Figura 5. Izquierda: pabellón del variómetro. Derecha: pabellón del magnetómetro escalar con efecto Overhauser. Ambos pabellones de madera son de dimensiones de $3 \times 3 \mathrm{~m}$.

Uno de los pabellones pequeños alberga el variómetro GSM-90FD5, y el otro pabellón el magnetómetro escalar GSM-90 EUROMAG, esto con la finalidad decomparar las mediciones entre los equipos. El pabellón más grande (Figura 6) es el destinado para albergar y realizar mediciones absolutas con el Bartington Mag-01H, y es el único de los pabellones que cuenta con dos pilares.

Para la construcción del observatorio geomagnético de Mérida fueron usados materiales no magnéticos, esto con la finalidad de que la construcción no generara perturbación a los instrumentos de mediciones. A pesar de esto, todos los materiales usados en la construcción fueron medidos previamente con un magnetómetro protónico para comprobar su perturbación o contaminación magnética (Jankowski y Sucksdorff, 1996).

Conjuntamente se construyeron dos pilares externos que permitirán realizar mediciones absolutas de la declinación "D" e inclinación "I" magnéticas eventualmente y adicionalmente dos marcas de acimut, necesarias como referencias para realizar las mediciones. En la Figura 7 se muestra uno de los pilares externos y una de las marcas de acimut.

\section{Sistema de comunicación y alimentación eléctrica del observatorio}

El sistema de alimentación eléctrica está constituido por un panel solar de 170 watt y $12 \mathrm{~V}$ DC con dos baterías de $12 \mathrm{~V}$ y $210 \mathrm{Ah}$ cada una. El panel solar y el banco de baterías fue la opción más conveniente como fuente de alimentación eléctrica para los equipos que conforman el observatorio, debido a que los pabellones se encuentran muy alejados de fuentes de energía eléctrica convencionales AC. El panel solar 


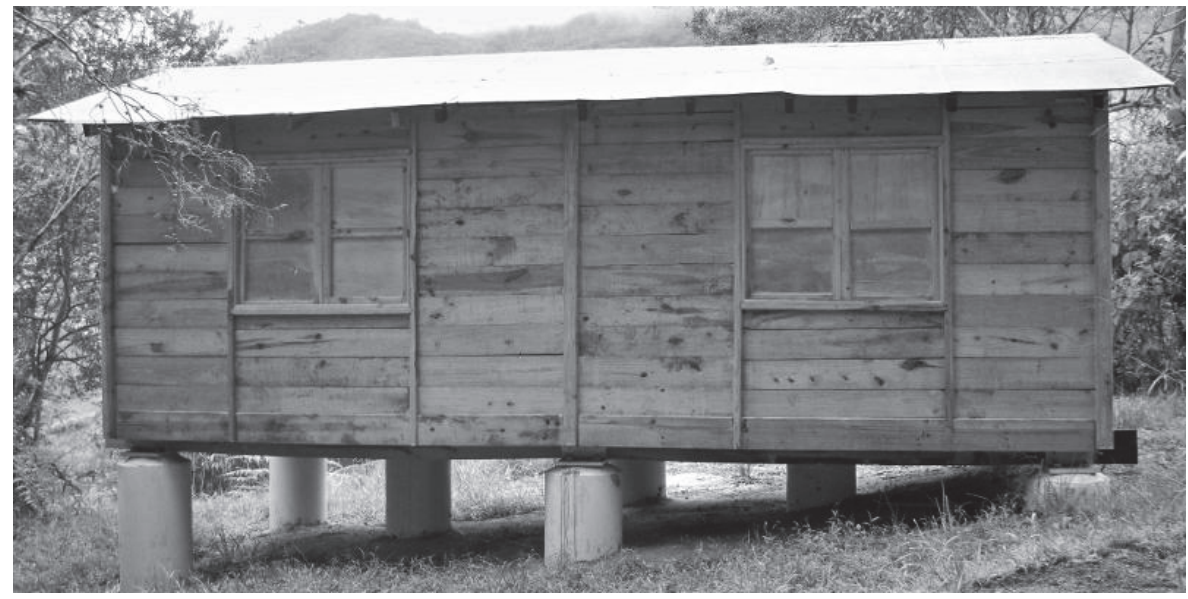

Figura 6. Pabellón para realizar mediciones absolutas, dimensiones de 3x6m.
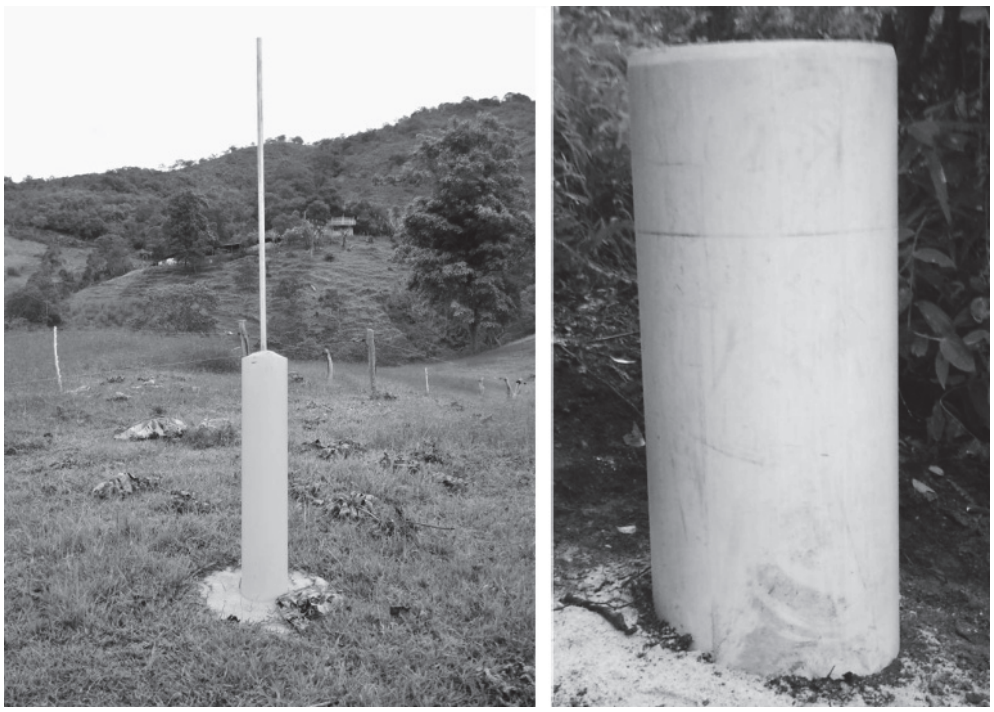

Figura 7. Izquierda: marca de acimut. Derecha: pilar externo.

y el banco de baterías fueron ubicados a una distancia de $100 \mathrm{~m}$ del pabellón del variómetro, para evitar perturbaciones.

Debido a la distancia y la topografía existente entre los pabellones del observatorio y la casa del IVIC-Mérida (Figura 2), se implementó un sistema de comunicación con radio modem serial para la transferencia y almacenamiento de los datos. 
Los datos son almacenados en una computadora que se encuentra en la casa del IVIC-Mérida. Entre las características que presenta el radio modem serial se tienen:

- Potencia: $1 \mathrm{~W}$

- Velocidad de transmisión: $115 \mathrm{kbbs}$

- Frecuencia de transmisión: 902-928Mhz

- Interfaces: Rs-232 / Rs-485

\section{Conclusiones}

Se logró la construcción del primer observatorio geomagnético en la República Bolivariana de Venezuela, consiguiendo alcanzar todos los objetivos planteados. El emplazamiento del mismo se encuentra en un área con gradientes magnéticos menores a $1 \mathrm{nT} / \mathrm{m}$, excelente para el funcionamiento del observatorio y favorable para el estudio del campo geomagnético, además libre de perturbaciones magnéticas tanto naturales como artificiales.

El observatorio, está conformado por instrumentos de mediciones (magnetómetros) adecuados para el estudio de campo geomagnético, y este observatorio cuenta con todos los requisitos iníciales para formar parte de la red INTERMAGNET.

El diseñado y construido de los pabellones que conforman el observatorio fueron realizados siguiendo recomendaciones internacionales. En el diseño se consideró la estabilidad de los pilares y de la temperatura (dentro de los pabellones). Ningún material empleado para la construcción del observatorio es magnético.

\section{Referencias}

Camacho E., Serra J., Muñiz M., Gandarrilla J. y Sira E., 2009. "Ubicación de un terreno para la construcción de un observatorio geomagnético", Memorias de la primera reunión Bienal de LATINMAG, Venezuela.

Gubbins D. y Herrero, E., 2007. "Encyclopedia of Geomagnetic and Paleomagnetism", Springer, Vol. 70, No. 1, pp. 61-66.

Jankowski J. y Sucksdorff C., 1996. Guide for magnetic measurements and observatory practice, publicado por la Asociación Internacional de Geomagnetismo y Aeronomía, Varsovia, pp. 36-50.

St-Louis B. y Sauter E., 2004. INTERMAGNET Technical Reference Manual. (2004). Versión 4.2. Descargado de la página: <www.intermagnet.org>.

Wienert K., 1970. Notes on geomagnetic observatory and survey practice, UNESCO, Belgium, pp. 15-23. 